Fabio Rocha Hoelz

\title{
Estabilidade de Escoamento de Couette sobre uma Parede Flexível
}

Dissertação apresentada como requisito parcial para obtenção do grau de Mestre pelo Programa de Pós-graduação em Engenharia de Fluidos do Departamento de Engenharia Mecânica da PUCRio

Orientador: Prof. Marcio da Silveira Carvalho 


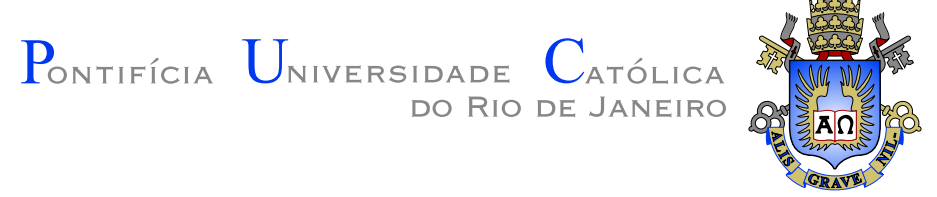

Fabio Rocha Hoelz

\section{Estabilidade de Escoamento de Couette sobre uma Parede Flexível}

Dissertação apresentada como requisito parcial para obtenção do grau de Mestre pelo Programa de Pós-graduação em Engenharia de Fluidos do Departamento de Engenharia Mecânica do Centro Técnico Científico da PUC-Rio. Aprovada pela Comissão Examinadora abaixo assinada.

Prof. Márcio da Silveira Carvalho

Orientador

Pontifícia Universidade Católica do Rio de Janeiro

Prof. André Augusto Isnard

Pontifícia Universidade Católica do Rio de Janeiro

Prof. Francisco Moura Neto

Universidade do Estado do Rio de Janeiro

Prof. José Eugenio Leal

Coordenador Setorial do Centro

Técnico Científico - PUC-Rio 
Todos os direitos reservados. É proibida a reprodução total ou parcial do trabalho sem autorização da universidade, do autor e do orientador.

Fabio Rocha Hoelz

Graduou-se em Engenharia Mecânica na Universidade do Estado do Rio de Janeiro - UERJ (Nova Friburgo, Brasil) em 2004.

Ficha Catalográfica

Hoelz, Fabio Rocha

Estabilidade de Escoamento de Couette sobre uma Parede Flexível / Fabio Rocha Hoelz; orientador: Marcio da Silveira Carvalho. - Rio de Janeiro : PUC-Rio, Departamento de Engenharia Mecânica, 2007.

v., 134 f: il. ; $29,7 \mathrm{~cm}$

1. Dissertação (mestrado) - Pontifícia Universidade Católica do Rio de Janeiro, Departamento de Engenharia Mecânica.

Inclui referências bibliográficas.

1. Engenharia Mecânica - Tese. 2. Estabilidade Hidrodinâmica. 3. Escoamento de Couette. 4. Escoamento com Superficies deformáveis. 5. Método dos Elementos Finitos. 6. Métodos Numéricos. I. Carvalho, Marcio da Silveira. II. Pontifícia Universidade Católica do Rio de Janeiro. Departamento de Engenharia Mecânica. III. Título. 


\section{Agradecimentos}

Ao meu orientador, Professor Márcio Carvalho que mesmo nos momentos mais difíceis depositou confiança em me trabalho e me deu completo apoio. E mesmo nos momentos em que parecia não haver solução não deixou de acreditar em mim.

Ao professores e pesquisadores André Isnard e Olderich Joel Romero, que em muito me ajudaram ao longo do desenvolvimento do meu trabalho, tirando duvidas e fazendo sugestões.

A todos os professores do Departamento de Engenharia Mecânica da PUC-Rio que com seus ensinamentos contribuiram para que eu chegasse aonde estou.

Ao corpo técnico-administrativo do DEM, pelas soluções dos meus problemas nos momentos mais críticos.

Aos meus pais e irmão por todo apoio, incentivo e carinho oferecidos durante esta caminhada, têm minha eterna gratidão.

A minha namorada Jaqueline da Silva Ferreira por toda a paciência com relação ao meu trabalho. Soube compreender minha omissão como namorado e abdicar de muitas coisas em meu favor.

A todos os amigos que fiz ao longo deste curso de mestrado que tornaram minha estadia nesta instutuição muito mais agradável: Eduardo, Ranena, Sully, Miguel, Victor Raul, Julio, Danmer, Juliana, Maria Angélica, Hugo, Sygifredo e Teresa.

E finalmente minha eterna gratidão ao CNPq e à PUC-Rio, pelos auxílios concedidos, sem os quais este trabalho não poderia ter sido realizado. 


\section{Resumo}

Hoelz, Fabio Rocha; Carvalho, Marcio da Silveira. Estabilidade de Escoamento de Couette sobre uma Parede Flexível. Rio de Janeiro, 2007. 134p. Dissertação de Mestrado — Departamento de Engenharia Mecânica, Pontifícia Universidade Católica do Rio de Janeiro.

Escoamentos de fluidos sobre paredes flexíveis se fazem presentes em diversos processos biológicos e industriais. A flexibilidade do sólido permite a propagação de ondas na interface, podendo levar o sistema a se tornar instável mesmo a baixos valores do número de Reynolds. Esta perda de estabilidade provoca uma alteração nas características hidrodinâmicas e na transferência de calor do processo. Os trabalhos disponíveis na literatura se concentram em torno de análise de estabilidade linear e experimentos de determinação de parâmetros críticos. Entretanto estas metodologias não são capazes de descrever o comportamento do sistema após sua desestabilização. Neste trabalho, o regime instável de um escoamento de Couette de um fluido Newtoniano sobre um sólido incompressível e impermeável de MooneyRivlin é estudado numericamente através da solução acoplada das equações de conservação de quantidade de movimento linear transiente de cada meio. O número de Reynolds foi escolhido pequeno o suficiente para afastar a possibilidade de que mecanismos inerciais se façam presentes. Diferentes razões de espessura líquido-sólido flexível foram utilizadas para se determinar os efeitos desta grandeza sobre o processo. O sistema de equações diferenciais foram integradas no espaço pelo método de Galerkin/elementos finitos, e no tempo por diferenças finitas. A necessidade de se utilizar passos de tempo variáveis exigiu o desenvolvimento de uma fórmula específica para a aproximação da derivada segunda presente no termo transiente do sólido.

\section{Palavras-chave}

Estabilidade Hidrodinâmica. Escoamento de Couette. Escoamento com Superficies deformáveis. Método dos Elementos Finitos. Métodos Numéricos. 


\section{Abstract}

Hoelz, Fabio Rocha; Carvalho, Marcio da Silveira. Couette Flow

Over a Flexible Wall Stability. Rio de Janeiro, 2007. 134p.

MsC Thesis - Department of Engenharia Mecânica, Pontifícia

Universidade Católica do Rio de Janeiro.

Fluid flow over flexible wall are present in several biological and industrial processes. The flexibility of the solid body permits the waves propagation along the interface, leading the system to become unstable even at low value of Reynolds number. This loss of stability induce some changes on the hydrodynamic characteristics and on the heat transfer of the process. Works available on literature are concentrated around linear stability analysis and experiments of determining critical parameters. Nevertheless these methodologies are not able of describing the system behavior after the desestabilization. In this work, the unstable regime of a Newtonian fluid Couette flow over an incompressible and impermeable Mooney-Rivlin solid is numerically studied by solving the coupled fluid and solid momentum equation. The Reynolds number has been chosen small enough to avoid the presence of inertial mechanisms. Different liquid-flexible solid thickness ratio were used to determine the effect of this parameter on the problem. The system of differential equations were integrated by Galerki's/finite elements method on space, and by finite differences on time. The necessity of using variables changeables time steps demanded the development of a specific equation to approximates the second material derivative present on the unsteady solid term.

\section{Keywords}

Hydrodynamic Stability. Couette Flow. Flow with Deformable Surface. Finite Elements Method. Numerical Methods. 


\section{Sumário}

$\begin{array}{lll}1 & \text { Introdução } & \mathbf{1 5}\end{array}$

\begin{tabular}{lll}
\hline 1.1 & Generalidades sobre Elastohidrodinâmica & 15
\end{tabular}

$\begin{array}{lll}1.2 & \text { Exposição do Problema } & 16\end{array}$

$\begin{array}{lll}1.3 & \text { Evidências Experimentais } & 19\end{array}$

\begin{tabular}{lll}
\hline 1.4 & Aplicações & 22
\end{tabular}

\begin{tabular}{lll}
\hline 1.5 & Objetivo & 23
\end{tabular}

1.6 Escopo e Roteiro da Dissertação 24

$\begin{array}{lll}2 & \text { Metodologia } & 25\end{array}$

$\begin{array}{lll}2.1 & \text { Configuração Geométrica } & 25\end{array}$

2.2 Metodologias e suas Limitações 27

\begin{tabular}{lll}
\hline 2.3 & Metodologia Aplicada & 29
\end{tabular}

3 Formulação Matemática 31

\begin{tabular}{lll}
\hline 3.1 & Modelagem do Fluido & 31
\end{tabular}

3.2 Solução do Problema de Fronteira Deformável 33

3.3 Geração Elíptica de Malhas 36

$\begin{array}{lll}3.4 & \text { Modelagem do sólido } & 39\end{array}$

$\begin{array}{lll}3.5 & \text { Acoplamento } & 47\end{array}$

$4 \quad$ Discretização das Equações 49

$4.1 \quad$ O Método de Galerkin/Elementos finitos 49

4.2 O Método das Diferenças Finitas 53

4.3 Expressões dos Resíduos das Equações 56

4.4 Solução do Sistema de Equações Não-lineares 59

5 O problema da Cavidade Inclinada $\quad 63$

$\begin{array}{lll}5.1 & \text { Introdução } & 63\end{array}$

5.2 Definição do Problema 65

$\begin{array}{lll}5.3 & \text { Metodologia } & 65\end{array}$

5.4 Definição de Parâmetros de Malha e do Fluido 68

$\begin{array}{lll}5.5 & \text { Resultados e Discussões } & 69\end{array}$

6 Validação da Rotina de Solução Transiente Fluido-Sólido 82

6.1 O Caso de Validação Estudado 83

$\begin{array}{lll}7 & \text { Resultados Numéricos } & \mathbf{9 0}\end{array}$

7.1 Teste de Malha e Parâmetros Críticos 92

7.2 Considerações da Sensibilidade do Sistema 104

$\begin{array}{lll}7.3 & \text { Resultados } & 113\end{array}$

$\begin{array}{lll}8 & \text { Conclusões e Sugestões } & 128\end{array}$

\begin{tabular}{lll}
\hline $8.1 \quad$ Conclusões e Comentários & 129
\end{tabular}

$\begin{array}{lll}8.2 & \text { Sugestões } & 131\end{array}$ 


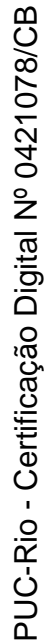




\section{Lista de figuras}

1.1 Escoamento de Couette de um fluido Newtoniano sobre um sólido rígido.

1.2 Linhas de corrente de um escoamento de Couette de um fluido Newtoniano sobre um sólido flexível $\Lambda<\Lambda_{\text {critic }}$.

1.3 Linhas de corrente de um escoamento de Couette de um fluido Newtoniano sobre um sólido flexível após a instabilidade.

1.4 Configuração dos experimentos realizados por Kumaran e Muralikrishnan, e Eggert e Kumar, utilizando um reômetro de placas paralelas.

1.5 Variação da viscosidade em função da taxa de cisalhamento ambos aparentes resultantes em um dos testes realizados por Kumaran. A linha contínua mostra o resultado para um escoamento entre superfíces rígidas.

1.6 Variação da viscosidade aparente ao longo do tempo quando a tensão de cisalhamento aplicada é mantida a um valor constante acima da tensão crítica em três testes realizados por Eggert e Kumar. 21

1.7 Variação da viscosidade aparente em função da taxa de cisalhamento de quatro testes realizados por Eggert e Kumar.

1.8 Variação da viscosidade ao longo do tempo quando a tensão de cisalhamento aplicada é elevada a um valor acima da crítica e então reduzida e mantida constante a um valor abaixo desta, em quatro testes realizados por Eggert e Kumar.

1.9 Cilindros concêntricos. O cilindro interno possui uma camada de um sólido flexível, enquanto o fluido permanece no espaço anular.

2.1 Seção transversal do cilindro do sistema de cilindros. 26

2.2 Variação linear da velocidade do cilindro externo.

3.1 Tipos de condições de contorno da equação de conservação de quantidade de movimento do fluido aplicadas nas fronteiras do domínio.

3.2 Domínio Físico do meio Fluido, parametrizado pelo vetor posição em coordenadas cartesianas $(x, y)$.

3.3 Domínio Computacional do meio Fluido, parametrizado pelo vetor $(\xi, \eta)$. 35

3.4 Localização das condições de contorno de malha na direção radial. 39

3.5 Localização das condições de contorno de malha na direção angular. 39

3.6 Mapeamento do domínio físico (deformado) no domínio de referência (não deformado).

3.7 Mapeamento do domínio físico (deformado) no domínio de referência (não deformado).

3.8 Balanço de Forças na interface sólido-fluido.

4.1 Elemento usado para discretizar o domínio fluido. Possui 9 nós e 39 graus de liberdade 
4.2 Elemento usado para discretizar o domínio sólido. Possui 9 nós e 21 graus de liberdade

4.3 Eixos ordenados $\varepsilon$ e $\vartheta$, que parametrizam as equações em cada elemento

5.1 Esquema da cavidade inclinada para o teste da rotina transiente.

5.2 Conjunto de soluções Vs. Número de Reynolds e tempo. A linha azul contínua representa as soluções em regime permanente, enquanto que a preta pontilhada representa as soluções encontradas pela rotina transiente ao longo do tempo.

5.3 Configuração geométrica e malha da cavidade inclinada.

5.4 Norma do campo de velocidade em função de $R e$ obtido por solução estacionária e continuação por pseudo-comprimento de arco.

5.5 Ampliação da região $B$ da figura 5.4

5.6 Ampliação da região D da figura 5.4.

5.7 Gráficos da norma da velocidade em função de Re obtidos por Pseudo-comprimento de arco e evolução temporal com perturbações de $\Delta R e=5$ no interior da região $\mathbf{B}$.

5.8 Gráficos da norma da velocidade em função de Re obtidos por Pseudo-comprimento de arco e evolução temporal com perturbações de $\Delta R e=20$ no interior da região $\mathbf{B}$.

5.9 Gráficos da norma da velocidade em função de $R e$ obtidos por Pseudo-comprimento de arco e evolução temporal com perturbações de $\Delta R e=50$ no interior da região $\mathbf{B}$.

5.10 Gráficos da norma da velocidade em função de $R e$ obtidos por Pseudo-comprimento de arco e evolução temporal com perturbações de $\Delta R e=100$ no interior da região $\mathbf{B}$.

5.11 Gráfico do Número de Reynolds crítico versus amplitude de perturbação do escoamento.

5.12 Gráficos da norma da velocidade em função de Re obtidos por Pseudo-comprimento de arco e evolução temporal com perturbações de $\Delta R e=10$ no interior da região $\mathbf{B}$.

5.13 Norma do vetor velocidade versus tempo do escoamento na cavidade logo após o sistema se tornar instável.

5.14 Espectro de freqüências do regime de escoamento instável na cavidade (amplitudes de perturbação iguais a 10).

5.15 Gráficos da norma da velocidade em função de Re obtidos por Pseudo-comprimento de arco e evolução temporal com perturbações de $\Delta R e=5$.

5.16 Gráficos da norma da velocidade em função de Re obtidos por Pseudo-comprimento de arco e evolução temporal com perturbações de $\Delta R e=50$.

5.17 Gráficos da norma da velocidade em função de $R e$ obtidos por Pseudo-comprimento de arco e evolução temporal com perturbações de $\Delta R e=100$.

6.1 Cavidade reta (quadrada), utilizada para testar a rotina transiente com acoplamento sólido-fluido. 
6.2 Relação Velocidade na parede superior Vs. tempo para cavidade revestida com sólido de Mooney-Rivlin.

6.3 Perfil de deformação dos meios sólido e fluido nos instantes 0,1 , $0,4,0,5$ e 2,0 para $R e=5$.

6.4 Perfil de deformação dos meios sólido e fluido no instantes 2,0 e regime permanente para $R e=5$.

86

6.5 Perfil de deformação dos meios sólido e fluido nos instantes 0,5 , $0,8,1,0$ e 2,6 para $R e=10$.

6.6 Perfil de deformação dos meios sólido e fluido nos instantes 0,6 , $1,4,2,0$ e 3,0 para $R e=20$.

6.7 Perfil de deformação dos meios sólido e fluido no instantes 2,6 e regime permanente para $R e=10$.

6.8 Perfil de deformação dos meios sólido e fluido no instantes 3,0 e regime permanente para $R e=20$.

7.1 Sistema sólido flexível - fluido com razão de espessuras igual a 1.

7.2 Sistema sólido flexível - fluido com razão de espessuras igual a 2.

7.3 Malha M1. A linha laranja indica a fronteira dos domínios (razão de espessuras igual à 1$)$.

7.4 Malha M2. A linha laranja indica a fronteira dos domínios (razão de espessuras igual à 1).

7.5 Malha M1 deformada (razão de espessuras igual a 1).

7.6 Malha M2 deformada (razão de espessuras igual a 1).

7.7 Gráfico representativo da norma da velocidade em função da razão de forças viscosas e elásticas de um escoamento sobre sólido flexível arbitrário em regime permanente.

7.8 Malha M3. A linha laranja indica a fronteira dos domínios (razão de espessuras igual a 1 ).

7.9 Malha M4. A linha laranja indica a fronteira dos domínios (razão de espessuras igual a 1).

7.10 Tensão de cisalhamento aplicada ao fluido em função do tempo.

7.11 Viscosidade aparente em função do tempo para tensão de cisalhamento controlada imposta ao fluido.

7.12 Velocidade aplicada na parede rígida em função do tempo.

7.13 Viscosidade aparente em função do tempo para velocidade da parede controlada.

7.14 Viscosidade aparente em função do tempo para velocidade da parede controlada.

7.15 Viscosidade aparente em função do tempo

7.16 Velocidade imposta na fronteira do domínio fluido devido movimento do cilindro externo em função do tempo

7.17 Viscosidade aparente em função do tempo para velocidade da parede controlada com $\Pi=1$.

7.18 Campo de velocidade do escoamento da metade superior do sistema no instante $t=4,2 s(\Pi=1)$.

7.19 Campo de velocidade do escoamento da metade superior do sistema no instante $t=5,3 s(\Pi=1)$.

7.20 Campo de velocidade do escoamento da metade superior do sistema no instante $t=6,0 s(\Pi=1)$. 
7.21 Campo de velocidade do escoamento da metade superior do sistema no instante $t=6,5 s(\Pi=1)$.

7.22 Perfil de deformações no instante $4,20 \mathrm{~s}$, para $\Pi=1$. 117

7.23 Perfil de deformações no instante $5,30 \mathrm{~s}$, para $\Pi=1$. 117

7.24 Perfil de deformações no instante $6,00 s$, para $\Pi=1$.

7.25 Perfil de deformações da metade superior dos domínios sólido e líquido no instante $t=6,50 \mathrm{~s}$, para sistema com $\Pi=1$.

7.26 Campo de pressões do escoamento no instante $t=6,40 \mathrm{~s}$, para sistema com $\Pi=1$.

7.27 Tensões sofridas pelo sólido durante a propagação de ondas. 119

7.28 Perfil de deformações no instante $6,50 \mathrm{~s}$, para $\Pi=1$. 121

7.29 Perfil de deformações no instante $6,60 \mathrm{~s}$, para $\Pi=1$. 121

7.30 Perfil de deformações no instante $6,70 s$, para $\Pi=1$. 121

7.31 Espectro de freqüências do escoamento instável $(\Pi=1)$. 122

7.32 Campo de velocidade do escoamento da metade superior do sistema no instante $t=6,5 s(\Pi=2)$. 123

7.33 Campo de velocidade do escoamento da metade superior do sistema no instante $t=8,5 s(\Pi=2)$.

7.34 Campo de velocidade do escoamento da metade superior do sistema no instante $t=9,5 s(\Pi=2)$.

7.35 Perfil de deformações no instante $6,50 \mathrm{~s}$, para $\Pi=2$. 124

7.36 Perfil de deformações no instante $8,50 \mathrm{~s}$, para $\Pi=2$. 124

7.37 Perfil de deformações no instante $9,50 \mathrm{~s}$, para $\Pi=2$.

7.38 Viscosidade aparente em função do tempo para velocidade da parede controlada com $\Pi=2$.

7.39 Perfil de deformações no instante $9,40 s$, para $\Pi=1$. 126

7.40 Perfil de deformações no instante $9,50 \mathrm{~s}$, para $\Pi=1$.

7.41 Perfil de deformações no instante $9,60 \mathrm{~s}$, para $\Pi=1$.

126

7.42 Espectro de freqüências do escoamento instável com razão de espessura $\Pi=2$. 


\section{Lista de tabelas}

7.1 Resultados obtidos com M1 e M2 95

$\begin{array}{lll}7.2 & \text { Valores de } \Lambda_{\text {crit }} \text { obtidos por continuação de ordem zero } & 97\end{array}$

7.3 Regime encontrado para cada $\Lambda$ imposto $(\Pi=1) \quad 99$

$\begin{array}{lll}7.4 & \text { Regime encontrado para cada } \Lambda \text { imposto }(\Pi=2) & 100\end{array}$

$\begin{array}{lll}7.5 & \text { Número de Reynolds e } \Lambda_{\text {crit }} \text { encontrados por método iterativo } & 100\end{array}$

7.6 Resultados obtidos para diversos valores de $\Delta t(\Pi=1) \quad 100$

7.7 Resultados utilizando malhas M2, M3 e M4 104

7.8 Parâmetros que caracterizam o sistema instável 104

7.9 Velocidade de propagação de ondas Vs. tempo $(\Pi=1) \quad 120$

7.10 Parâmetros que caracterizam o sistema instável $(\Pi=2) \quad 122$

7.11 Velocidade de propagação de ondas Vs. tempo $(\Pi=2) \quad 125$ 
Uma poderosa ferramenta para nos ajudar a gerir com habilidade a nossa vida é perguntar antes de cada ato se isso no trará felicidade. isso vale desde a hora de decidir se vamos ou não usar drogas até se vamos ou não comer aquele terceiro pedaço de torta de banana com creme

Dalai Lama. 